\title{
Abundance of barium in the atmospheres of red giants in the Galactic globular cluster NGC 104 (47 Tuc) ${ }^{\star}$
}

\author{
V. Dobrovolskas ${ }^{1}$, E. Kolomiecas ${ }^{1}$, A. Kučinskas ${ }^{1}$, J. Klevas ${ }^{1}$, and S. Korotin ${ }^{2}$ \\ 1 Institute of Theoretical Physics and Astronomy, Vilnius University, Sauletekio al. 3, Vilnius, LT-10257, Lithuania \\ e-mail: vidas.dobrovolskas@tfai.vu.lt \\ ${ }^{2}$ Crimean Astrophysical Observatory, Nauchny 298409, Crimea \\ Received ; accepted
}

\section{ABSTRACT}

\begin{abstract}
Context. While most (if not all) Type I Galactic globular clusters (GGCs) are characterised by spreads in the abundances of light chemical elements (e.g. $\mathrm{Li}, \mathrm{N}, \mathrm{O}, \mathrm{Na}, \mathrm{Mg}, \mathrm{Al}$ ), it is not yet well established whether similar spreads may exist in s-process elements as well.

Aims. We investigated the possible difference in Ba abundance between the primordial (1P) and polluted (2P) stars in the Galactic globular cluster (GGC) 47 Tuc (NGC 104). For this, we obtained homogeneous abundances of Fe, Na, and Ba in a sample of 261 red giant branch (RGB) stars which is the largest sample used for Na and Ba abundance analysis in any GGC so far.

Methods. Abundances of $\mathrm{Na}$ and $\mathrm{Ba}$ were determined using archival GIRAFFE/VLT spectra and 1D non-local thermodynamic equilibrium (NLTE) abundance analysis methodology.

Results. Contrary to the finding of Gratton et al. (2013), we did not detect any significant Ba-Na correlation or 2P-1P Ba abundance difference in the sample of 261 RGB stars in 47 Tuc. This corroborates the result of D'Orazi et al. (2010) who found no statistically significant $\mathrm{Ba}-\mathrm{Na}$ correlation in $110 \mathrm{RGB}$ stars in this GGC. The average barium-to-iron ratio obtained in the sample of $261 \mathrm{RGB}$ stars, $\left\langle[\mathrm{Ba} / \mathrm{Fe}]_{1 \mathrm{D} \text { NLTE }}\right\rangle=-0.01 \pm 0.06$, agrees well with those determined in Galactic field stars at this metallicity and may therefore represent the abundance of primordial proto-cluster gas that has not been altered during the subsequent chemical evolution of the cluster.
\end{abstract}

Key words. Techniques: spectroscopic - Stars: abundances - Stars: late-type - globular clusters: individual

\section{Introduction}

Most if not all Galactic globular clusters contain multiple stellar populations which are characterised by different abundances of chemical elements, radial distributions in the given GGC, and kinematic properties (see e.g. Bastian \& Lardo 2018, for a review). It is usually assumed that a fraction of the GGC stars, so-called second-generation stars (2P), were enriched in certain chemical elements and depleted in others by some first-generation (1P) polluters. A number of enrichment scenarios have been proposed to explain the observed properties of the GGCs by involving various potential polluters, such as fast-rotating massive stars (FRMS; e.g., Decressin et al. 2007), asymptotic giant branch (AGB) stars (e.g., D’ Antona et al. 2016), super-massive stars (SMS, $10^{4} \mathrm{M}_{\odot}$; e.g., Denisenkov \& Hartwick 2014; Gieles et al. 2018). Unfortunately, none of the proposed scenarios is capable of explaining chemical and kinematic differences between the $1 \mathrm{P}$ and 2P stars simultaneously (Bastian \& Lardo 2018; Gratton et al. 2019).

The $1 \mathrm{P}-2 \mathrm{P}$ differences in the abundances of chemical elements have been detected as correlations or anti-correlations between the abundances of light elements: Na-Li (Bonifacio et al. 2007) correlation and $\mathrm{O}-\mathrm{Na}, \mathrm{Mg}-\mathrm{Al}$ (Carretta et al. 2009), Li-O (Pasquini et al. 2005; Shen et al. 2010) anti-correlations. Except

\footnotetext{
$\star$ Based on observations obtained at the European Southern Observatory (ESO) Very Large Telescope (VLT) at Paranal Observatory, Chile.
}

for the so-called Type II GGCs (see Marino et al. 2015, 2019 for details), the majority of Galactic GGCs (Type I) seem to be uniform in their Fe-group, as well as s- and r-process element abundances.

As for the s-process elements, results of several studies have suggested that there may be some variation in their abundances in several Type I GGCs, too. For example, Gratton et al. (2013) reported a possible $\mathrm{Na}-\mathrm{Ba}$ correlation in the sample of 106 red horizontal branch (RHB) stars in NGC 104 (47 Tuc). While statistical significance of the possible correlation was found to be high, the authors have warned that, because of a small range in the $[\mathrm{Ba} / \mathrm{Fe}]$ variation and $[\mathrm{Na} / \mathrm{O}]$ correlation with the effective temperature along the horizontal branch (HB), their result needed to be confirmed in further studies for claiming a definite detection (Gratton et al. 2013). Some other studies have shown tentative signs for a possible correlation between the abundances of light and s-process elements in other Type I GGCs, such as signatures for $\mathrm{Sr}-\mathrm{Na}$ and $\mathrm{Y}-\mathrm{Na}$ relations in M 4 (Spite et al. 2016; Villanova \& Geisler 2011; but see also D’Orazi et al. 2013). Also, our recent analysis of $\mathrm{Zr}$ abundance in 237 RGB stars in 47 Tuc suggests the existence of weak but statistically significant $\mathrm{Na}-\mathrm{Zr}$ correlation and $2 \mathrm{P}-1 \mathrm{P} \mathrm{Zr}$ abundance difference of $\Delta[\mathrm{Zr} / \mathrm{Fe}]_{2 \mathrm{P}-1 \mathrm{P}} \approx 0.06$ Kolomiecas et al. 2021). The existence of such correlations would indicate that the light and s-process elements should have been produced in the same polluters that have enriched the $2 \mathrm{P}$ stars in some elements and depleted in others. Unfortunately, the data obtained so far is 
inconclusive thus further analysis of more s-process elements in the larger samples of GGC stars would be desirable.

With an aim of shedding more light on the possible 1P-2P differences in s-process element abundances, in this study we determined Ba abundances in 261 RGB stars in 47 Tuc, a Type I GGC (Marino et al. 2019). To the best of our knowledge, this is the only GGC in which a tentative detection of statistically significant relation between the light and s-process element abundances has been reported. Therefore, our primary goal was to verify whether the $\mathrm{Na}-\mathrm{Ba}$ correlation and/or 1P-2P Ba abundance difference could be detected in our RGB star sample which is the largest one in which $\mathrm{Ba}$ abundance was determined in any GGC so far.

The paper is structured as follows: after a short introduction we provide a brief description of the observational data (Sect.2) and abundance determination methodology (Sect. 3). The obtained results are discussed in Sect. 4 and the main findings are summarized in the Conclusions.

\section{Observational data}

In this work we studied a sample of RGB stars using the highresolution spectra that were obtained with GIRAFFE/VLT during three observing programs, 072.D-0777(A), PI: François; 073.D0211(A), PI: Carretta; and 088.D-0026(A), PI: McDonald (Table (1). Individual spectra were retrieved for the analysis from the ESO Advanced Data Products Archive1. Our stellar sample overlaps with that utilized by Kolomiecas et al. (2021) in their study of $\mathrm{Zr}$ in 47 Tuc (228 stars in common) but it also includes 33 additional targets that were not analysed in Kolomiecas et al. (2021).

Median-averaged sky spectrum was obtained from the dedicated sky fibres and subsequently was subtracted from the individual spectra of all target stars. In the case of the program 088.D-0026(A) where three exposures for the same targets were available, spectra were co-added to increase signal-to-noise ratio, $S / N$, with the latter determined at the continuum level in the vicinity of the investigated $\mathrm{Na}$ I and $\mathrm{Ba}$ II lines $(\sim 619.7 \mathrm{~nm})$. The $S / N$ ratio of the final spectra varied from $S / N \sim 220$ for the targets at $T_{\text {eff }} \sim 4200 \mathrm{~K}$ to $S / N \sim 60$ at $T_{\text {eff }} \sim 4700 \mathrm{~K}$. All target spectra were continuum normalized using the splot task under IRAF (Tody 1986). Radial velocities were determined using the fxcor task in IRAF and cross-correlation technique. For this, we used synthetic spectrum computed with the model atmosphere having $T_{\text {eff }}=4500 \mathrm{~K}, \log g=1.90$, and $[\mathrm{Fe} / \mathrm{H}]=-0.7$ to represent a typical RGB star in 47 Tuc. The obtained radial velocities were then used to shift the wavelengths to the rest frame with the dopcor task in IRAF.

Only RGB stars were selected for the abundance analysis, by discarding $\mathrm{HB}$ and AGB stars based on their position in the $V-(V-I)$ colour magnitude diagram. Before performing abundance analysis, we verified that all targets are members of 47 Tuc which was done based on their proper motions taken from the Gaia EDR3 catalogue (Gaia Collaboration et al. 2021). Following Milone et al. (2018), we required that the proper motions of the cluster member stars did not deviate by more

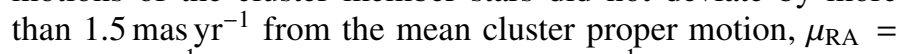
$5.25 \mathrm{mas} \mathrm{yr}^{-1}$ and $\mu_{\mathrm{DEC}}=-2.53 \mathrm{mas} \mathrm{yr}^{-1}$ Baumgardt et al. 2019).

Stars common to several programs were identified by crossmatching their coordinates within the 1 arcsec radius. This resulted in 14 stars in common to the 073.D-0211(A) and

\footnotetext{
${ }^{1}$ http://archive.eso.org/wdb/wdb/adp/phase3_spectral/form
}

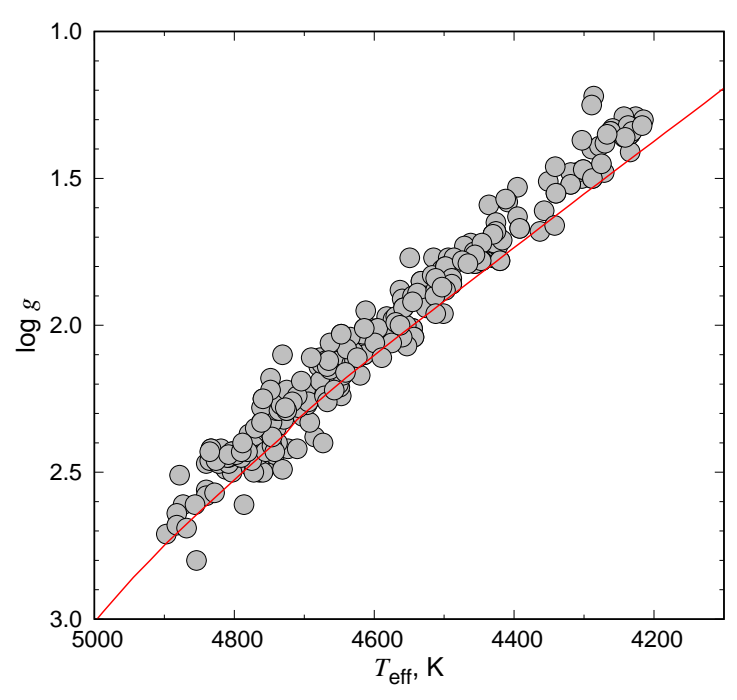

Fig. 1. Target RGB stars in the $T_{\text {eff }}-\log g$ plane. The red line is YonseiYale (Kim et al. 2002) isochrone $(12 \mathrm{Gyr},[\mathrm{M} / \mathrm{H}]=-0.68,[\alpha / \mathrm{Fe}]=$ $+0.4)$.

088.D-0026(A) samples, 23 stars to 072.D-0777(A) and 088.D0026(A), 17 stars to 072.D-0777(A) and 073.D-0211(A), and 5 stars in common to all three samples (Table A.1). Abundances of these stars were computed by averaging those obtained using spectra of individual observing programs.

The final target sample consisted of 261 unique RGB stars for which we determined $\mathrm{Fe}, \mathrm{Na}$, and $\mathrm{Ba}$ abundances. Our RGB sample is therefore somewhat larger than that of Kolomiecas et al. 2021, 237 objects) because it includes stars with $T_{\text {eff }}>4800 \mathrm{~K}$ which were discarded from their analysis.

\subsection{Atmospheric parameters}

Atmospheric parameters for the majority of targets (228 stars) were determined by Kolomiecas et al. (2021). For the remaining 33 stars we used the same procedure as utilised by the latter authors. With this approach, effective temperatures were determined using photometry from Bergbusch \& Stetson (2009) and color- $T_{\text {eff }}$ calibration of Ramirez \& Melendez (2005). The obtained values were verified by checking trends in the iron abundance - lower excitation potential plane. In most cases, the slopes were consistent with zero thereby suggesting a good agreement of photometric and spectroscopic $T_{\text {eff }}$ values.

Surface gravities were obtained by using a classical relation between stellar mass, luminosity, effective temperature, and surface gravity. This decision was made because only a few Fe II lines were available in the stellar spectra (typically, 1-4 lines). To compute gravities from photometry, we assumed an identical mass of $0.87 \mathrm{M}_{\odot}$ for all RGB stars investigated in this work, as indicated by the Yonsei-Yale isochrone of $12 \mathrm{Gyr}$ and $[\mathrm{M} / \mathrm{H}]=-0.68$ (Kim et al. 2002). Bolometric luminosities were computed using relation between the bolometric correction, $T_{\mathrm{eff}}$, and metallicity from Alonso et al. (1999).

Microturbulence velocities were obtained by enforcing zero trend in the iron abundance - equivalent width plane while keeping photometric effective temperature fixed. In this procedure strong lines were neglected $(W>15 \mathrm{pm})$ because of their reduced sensitivity to microturbulence velocity. Due to small number (17-28) of iron lines, the estimated accuracy of microturbulence velocity was $\approx 0.2 \mathrm{~km} \mathrm{~s}^{-1}$ and represents the main source of uncertainty in the determined $\mathrm{Ba}$ abundances (Section 3.5). 
Table 1. Spectroscopic data used in this work.

\begin{tabular}{ccccccc}
\hline \hline Programme & $\begin{array}{c}\text { Date of } \\
\text { observations }\end{array}$ & Setting & $\begin{array}{c}\lambda_{\text {central, }} \\
\mathrm{nm}\end{array}$ & $\mathrm{R}$ & $\begin{array}{c}\text { Exposure, } \\
\mathrm{s}\end{array}$ & $\begin{array}{c}\text { Number of } \\
\text { stars }\end{array}$ \\
\hline 072.D-0777(A) & $2003-10-21$ & HR13 & 627.3 & 22500 & 1500 & 112 \\
& $2003-10-21$ & HR13 & 627.3 & 22500 & 3600 & 121 \\
073.D-0211(A) & $2004-07-07$ & HR13 & 627.3 & 22500 & 1600 & 113 \\
088.D-0026(A) & $2011-11-26$ & HR13 & 627.3 & 22500 & $3 \times 700$ & 113 \\
& $2011-11-26$ & HR14A & 651.5 & 17740 & $3 \times 340$ & 113 \\
\hline
\end{tabular}

Table 2. Atomic parameters of $\mathrm{Na}$ I and $\mathrm{Ba}$ II lines used in this study.

\begin{tabular}{lllllll}
\hline \hline Element & $\lambda, \mathrm{nm}$ & $\chi, \mathrm{eV}$ & $\log g f^{\mathrm{a}}$ & $\log \gamma_{\text {rad }} \mathrm{b}$ & $\log \frac{\gamma_{4} \mathrm{c}}{N_{e}}$ & $\log \frac{\gamma_{6} \mathrm{~d}}{N_{H}}$ \\
\hline $\mathrm{Na}_{\mathrm{I}}$ & 615.4225 & 2.102 & -1.547 & 7.85 & -4.39 & -7.28 \\
$\mathrm{Na}_{\mathrm{I}}$ & 616.0747 & 2.104 & -1.246 & 7.85 & -4.39 & -7.28 \\
$\mathrm{Ba}_{\text {II }}$ & 614.1730 & 0.704 & -0.076 & 8.20 & -5.48 & -7.47 \\
$\mathrm{Ba}_{\text {II }}$ & 649.6910 & 0.604 & -0.377 & 8.10 & -5.48 & -7.47 \\
\hline
\end{tabular}

a Wiese \& Martin (1980); $;{ }^{\mathrm{b}}$ Mashonkina \& Bikmaev (1996); ${ }^{\mathrm{c}}$ Kupka et al. (2000); ${ }^{\mathrm{d}}$ Korotin et al. (2011)

The location of the target RGB stars in the $T_{\text {eff }}-\log g$ plane is shown in Fig. 11 Atmospheric parameters of the individual target stars are listed in Table D.1.

\section{Abundance analysis}

Because the stellar sample used in the present study overlaps with that investigated by Kolomiecas et al. (2021), abundances of $\mathrm{Fe}$ and $\mathrm{Na}$ in targets common to the two studies (228 stars) were taken from Kolomiecas et al. (2021). In addition, we determined $\mathrm{Fe}$ and $\mathrm{Na}$ abundances in $33 \mathrm{RGB}$ stars and $\mathrm{Ba}$ abundances in 261 stars. In both studies abundance analysis of $\mathrm{Fe}$ and $\mathrm{Na}$ was performed following strictly identical procedures. Abundances of $\mathrm{Na}$ and $\mathrm{Ba}$ were determined using ATLAS9 model atmospheres (Kurucz 1993; Sbordone et al. 2004; Sbordone 2005) and spectral synthesis, under the assumption of non-local thermodynamic equilibrium (NLTE), while local thermodynamic equilibrium (LTE) was assumed in the analysis of Fe. Procedures involved in the abundance determination are described below.

\subsection{Reference abundances in the Sun}

Reference abundances in the Sun were determined using the Kitt Peak Solar Flux atlas (Kurucz et al. 1984). To briefly summarize, Fe abundance was obtained using the equivalent width method, with the latter determined by fitting the Voigt profiles to the observed spectral line profiles. The Fe I and Fe II line list used for abundance determination is provided in Table B.1 Oscillator strength data were obtained from the VALD3 database (Ryabchikova et al. 2015).

Microturbulence velocity was obtained by iteration until there was zero trend of iron abundance with the line strength. Our determined value of $\xi_{\text {micro }}=0.93 \mathrm{~km} \mathrm{~s}^{-1}$ is in good agreement with the typical value of $0.9-1.0 \mathrm{~km} \mathrm{~s}^{-1}$ available in the literature (Doyle et al. 2014). The average solar iron abundance obtained with the determined microturbulence velocity was $A(\mathrm{Fe})=7.55 \pm 0.01(\sigma=0.06$; here \pm 0.01 is the error of the mean, $\sigma$ denotes standard deviation due to the line-toline abundance variation) which was obtained from 29 lines with $W<10.5 \mathrm{pm}$ (Table B.1). Although the scatter is significant, the average abundance is in good agreement with those available in the literature, e.g. $A(\mathrm{Fe})=7.51 \pm 0.06$ from Caffau et al. (2011).

For the solar $\mathrm{Na}$ abundances, we used $A(\mathrm{Na})=6.17$ which was determined with the NLTE spectral line synthesis methodology (Sect. 3.3) and by using the same $\mathrm{Na}$ I lines as utilised in the analysis of the target stars in 47 Tuc. This value agrees well with those obtained in the earlier studies, e.g. $A(\mathrm{Na})_{\odot}=6.21 \pm 0.04$ derived by Scott et al. (2015a).

Solar abundance of $\mathrm{Ba}$ was obtained by utilising the NLTE methodology (Sec. 3.4). The determined value, A(Ba) $=2.17$, agrees reasonably well with $A(\mathrm{Ba})_{1 \mathrm{D} \text { NLTE }}=2.07$ and $A(\mathrm{Ba})_{3 \mathrm{D} \text { NLTE }}=2.28$ derived by Gallagher et al. (2020) and coincides with the meteoritic value of $2.17 \pm 0.02$ from Lodders (2021).

\subsection{Determination of $\mathrm{Fe}$ abundance}

Abundances of $\mathrm{Fe}$ in the target RGB stars were obtained using a set of $17-28 \mathrm{Fe}_{\mathrm{I}}$ lines $(612.79-691.67 \mathrm{~nm})$, with the line lower level excitation potentials in the range of $2.18-4.61 \mathrm{eV}$ (Table B.1). The mean value obtained in the sample of 261 stars was $\langle[\mathrm{Fe} / \mathrm{H}]\rangle=-0.75 \pm 0.05$ (the error denotes standard deviation due to the star-to-star abundance scatter) which agrees well with the values determined in the earlier studies (cf. $\langle[\mathrm{Fe} / \mathrm{H}]\rangle=-0.74 \pm 0.05$, Carretta et al. 2009, 114 RGB stars). The Fe abundances obtained in 261 target RGB stars are listed in Table D.1.

\subsection{Determination of $\mathrm{Na}$ abundance}

Two Na I lines located at $615.42 \mathrm{~nm}$ and $616.07 \mathrm{~nm}$ were used in the determination of $\mathrm{Na}$ abundances (Table 2). The model atom of $\mathrm{Na}$ used in this work was the same as described in Dobrovolskas et al. (2014). It consisted of the first 20 energy levels of $\mathrm{Na}$ I and the ground level of $\mathrm{Na}$ II, with a total of 46 radiative transitions taken into account. The rate coefficients of collisions with hydrogen atoms for the lower 9 levels of $\mathrm{Na}$ I were taken from Barklem et al. (2010). Examples of the bestfitted $\mathrm{Na}$ I line profiles in the Na-poor and Na-rich stars are shown in Fig. 2, while the $\mathrm{Na}$ abundances determined in all target stars are plotted in the middle row of Fig. 4 and are 



Fig. 2. Examples of the observed (black dots) and best-fitted synthetic Na I line profiles (red lines) in the GIRAFFE spectra of the target stars characterised by different $\mathrm{Na}$ abundances and effective temperatures ( $T_{\text {eff }} \approx 4250 \mathrm{~K}$, top row; $T_{\text {eff }} \approx 4750 \mathrm{~K}$, bottom row). The continuum level is shown as the gray dashed line.
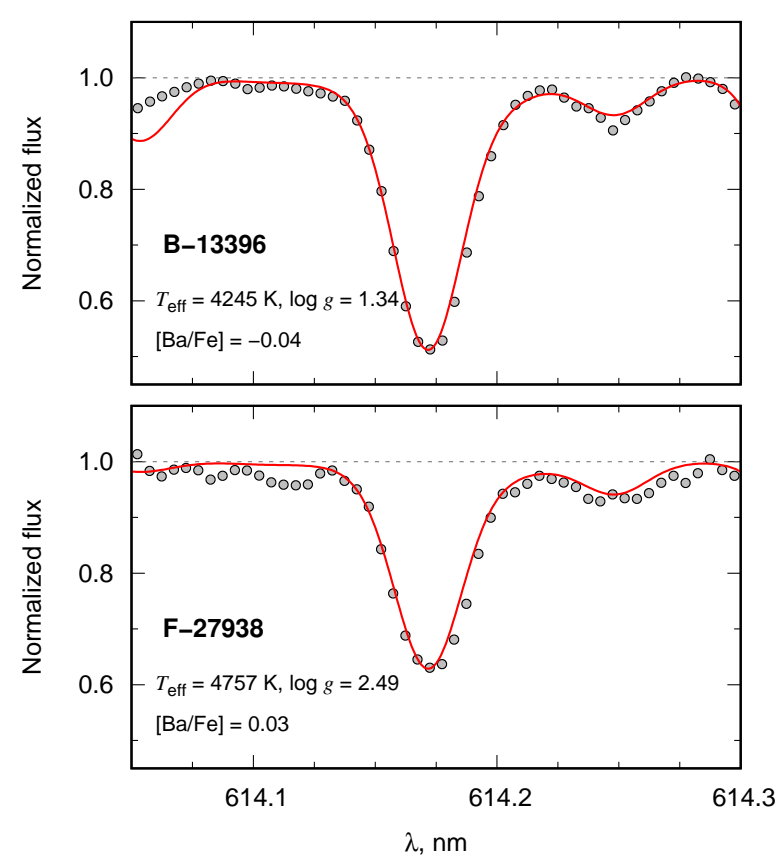

Fig. 3. Examples of the observed (black dots) and best-fitted synthetic spectra in the vicinity of Ba II lines (red lines) in the GIRAFFE spectra of the target stars characterised by different effective temperatures ( $T_{\text {eff }} \approx 4250 \mathrm{~K}$, top panel; $T_{\text {eff }} \approx 4750 \mathrm{~K}$, bottom panel). The continuum level is shown as the gray dashed line.

listed in Table D.1 The Na NLTE-LTE abundance corrections, $\Delta_{1 \mathrm{DNLTE}-\mathrm{LTE}}=A(\mathrm{Na})_{1 \mathrm{DNLTE}}-A(\mathrm{Na})_{1 \mathrm{DLTE}}$, were in the range $-0.07 \ldots-0.27$, depending on the strength of the spectral line and effective temperature of the target star (Table 3).
Table 3. 1D NLTE-LTE abundance corrections for Na and Ba.

\begin{tabular}{|c|c|c|c|}
\hline Element & $\lambda, \mathrm{nm}$ & $W, \mathrm{pm}$ & $\Delta_{1 \text { D NLTE-LTE }}$ \\
\hline \multicolumn{4}{|c|}{$T_{\text {eff }}=4250 \mathrm{~K}, \log g=1.35$} \\
\hline \multicolumn{4}{|c|}{ Na-poor } \\
\hline $\mathrm{Na}$ I & 615.42 & 5.7 & -0.08 \\
\hline $\mathrm{Na}$ I & 616.07 & 8.7 & -0.14 \\
\hline Ba II & 614.17 & 19.0 & -0.06 \\
\hline \multicolumn{4}{|l|}{$\mathrm{Na}$-rich } \\
\hline $\mathrm{Na}$ I & 615.42 & 9.6 & -0.19 \\
\hline $\mathrm{Na}$ I & 616.07 & 11.8 & -0.27 \\
\hline \multicolumn{4}{|c|}{$T_{\text {eff }}=4750 \mathrm{~K}, \log g=2.40$} \\
\hline \multicolumn{4}{|c|}{ Na-poor } \\
\hline $\mathrm{Na}$ I & 615.42 & 3.6 & -0.07 \\
\hline $\mathrm{Na}$ I & 616.07 & 5.4 & -0.08 \\
\hline Ba II & 614.17 & 14.8 & -0.10 \\
\hline \multicolumn{4}{|l|}{ Na-rich } \\
\hline $\mathrm{Na}$ I & 615.42 & 6.3 & -0.12 \\
\hline $\mathrm{Na}$ I & 616.07 & 8.7 & -0.15 \\
\hline
\end{tabular}

\subsection{Determination of $\mathrm{Ba}$ abundance}

As in the case of $\mathrm{Na}$, abundances of $\mathrm{Ba}$ were determined using spectral synthesis approach, under the assumption of NLTE. Two Ba II lines located at $614.17 \mathrm{~nm}$ and $649.69 \mathrm{~nm}$ were used for the analysis whenever possible though in most cases only the $614.17 \mathrm{~nm}$ line was available for the analysis (Table 2). The level departure coefficients and spectral line profiles were computed using the version of MULTI code (Carlsson 1986) modified by (Korotin et al. 1999). To take into account the fact that $614.17 \mathrm{~nm}$ line is blended with Fe I line, the spectra were computed with the SynthV code (Tsymbal 1996). For this, we used NLTE departure coefficients of Ba computed with the MULTI code while the Fe I line was synthesized under the assumption of LTE. The model atom of Ba used in this work was the same as described in Andrievsky et al. (2009). It consisted of 31 levels 


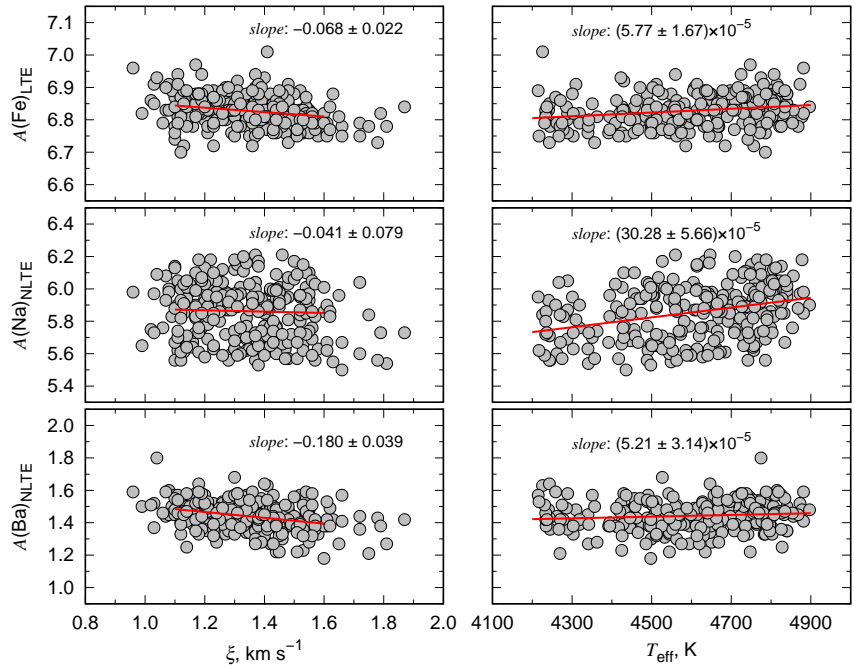

Fig. 4. Fe (top row), $\mathrm{Na}$ (middle row), and $\mathrm{Ba}$ (bottom row) abundances plotted against the microturbulence velocity (left column) and effective temperature (right column). In all panels the linear fits to the data are shown as the solid red lines.

Table 4. Typical Fe, $\mathrm{Na}$, and $\mathrm{Ba}$ abundance measurement errors.

\begin{tabular}{ccccccc}
\hline \hline Element & $\begin{array}{c}\sigma\left(T_{\text {eff }}\right) \\
\text { dex }\end{array}$ & $\begin{array}{c}\sigma(\log g) \\
\text { dex }\end{array}$ & $\begin{array}{c}\sigma\left(\xi_{\mathrm{t}}\right) \\
\text { dex }\end{array}$ & $\begin{array}{c}\sigma(\text { cont }) \\
\text { dex }\end{array}$ & $\begin{array}{c}\sigma(\text { fit }) \\
\text { dex }\end{array}$ & $\begin{array}{c}\sigma_{A(\mathrm{X})}(\text { total }) \\
\text { dex }\end{array}$ \\
\hline \multicolumn{7}{c}{$T_{\text {eff }}=4250 \mathrm{~K}, \log g=1.35$} \\
$\mathrm{Fe}$ & 0.03 & 0.02 & 0.12 & 0.10 & 0.02 & 0.16 \\
$\mathrm{Na}$ & 0.06 & 0.01 & 0.05 & 0.03 & 0.04 & 0.09 \\
$\mathrm{Ba}$ & 0.01 & 0.04 & 0.10 & 0.04 & 0.03 & 0.12 \\
& \multicolumn{7}{c}{$T_{\text {eff }}=4750 \mathrm{~K}, \log g=2.40$} \\
$\mathrm{Fe}$ & 0.04 & 0.02 & 0.11 & 0.10 & 0.05 & 0.16 \\
$\mathrm{Na}$ & 0.06 & 0.01 & 0.03 & 0.03 & 0.05 & 0.09 \\
$\mathrm{Ba}$ & 0.02 & 0.02 & 0.08 & 0.05 & 0.06 & 0.12 \\
\hline
\end{tabular}

of Ba I, 101 levels of Ba II $(n<50)$, and the ground level of Ba III. In total, 91 bound-bound transitions between the lowest 28 energy levels $(n<12, l<5)$ of Ba II were taken into account. Fine structure was included for the levels $5 \mathrm{~d}^{2} \mathrm{D}$ and $6 \mathrm{p} 2 \mathrm{P}^{0}$, according to the prescription given in Andrievsky et al. (2009). Following the latter study, the hyperfine structure of the $649.69 \mathrm{~nm}$ line was approximated using three components (lines).

Only the 088.D-0026(A) sample contained spectra where both $\mathrm{Ba}$ II lines were available for every target star. In this case, the final $\mathrm{Ba}$ abundance value was taken as an average of estimates obtained from the two lines. In all other cases Ba abundance was determined using a single $614.17 \mathrm{~nm}$ line. Since Ba II lines are strong, we did not take into account their possible contamination by $\mathrm{CN}$ lines and their influence on the Ba abundance determination. We nevertheless estimate that this effect should not exceed 0.01 dex. The obtained $\mathrm{Ba}$ abundances are shown in the bottom row of Fig. 4 and are listed in Table D.1 Typical examples of the 1D NLTE synthetic spectrum fits to the Ba II $614.17 \mathrm{~nm}$ spectral line in the target stars characterised by different effective temperatures are provided in Fig. 3. The $\Delta_{1 D N L T E-L T E}$ abundance corrections for $\mathrm{Ba}$ were in the range $-0.06 \ldots-0.10$, increasing in magnitude at higher effective temperatures (Table 3).

The average barium-to-iron abundance ratio determined in the sample of 261 was $\left\langle[\mathrm{Ba} / \mathrm{Fe}]_{1 \mathrm{D} \text { NLTE }}\right\rangle=-0.01 \pm 0.06$ where the error is standard deviation due to star-to-star abundance variation.

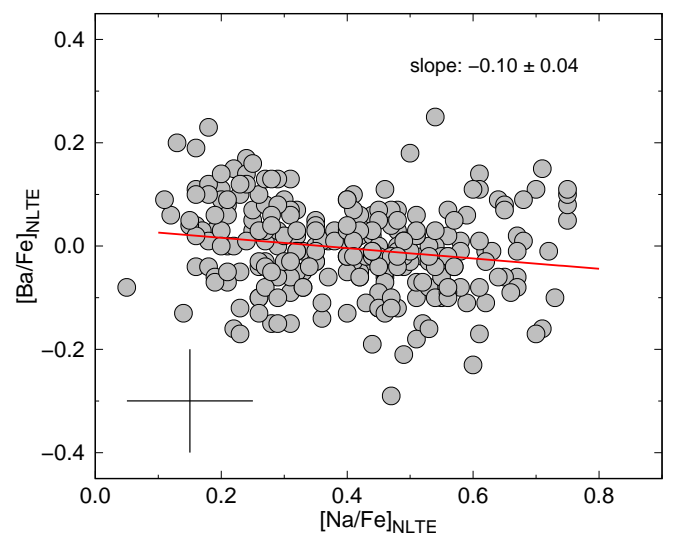

Fig. 5. $[\mathrm{Ba} / \mathrm{Fe}]$ abundance ratios determined in $261 \mathrm{RGB}$ stars in $47 \mathrm{Tuc}$, plotted versus their $[\mathrm{Na} / \mathrm{Fe}]$ abundance rations. Typical abundance error bars are shown in the bottom left corner. The linear fit to the data is shown as the solid red line.

\subsection{Errors in the determined $\mathrm{Fe}, \mathrm{Na}$, and $\mathrm{Ba}$ abundances}

Uncertainties in the determined $\mathrm{Fe}, \mathrm{Na}$, and $\mathrm{Ba}$ abundances were determined using the prescription given in Cerniauskas et al. (2018), by utilising ATLAS9 models computed with $T_{\text {eff }}=$ $4250 \mathrm{~K}, \log g=1.35$; and $T_{\text {eff }}=4750 \mathrm{~K}, \log g=2.40$. In essence, we first estimated errors in the effective temperature, surface gravity, microturbulence velocity, continuum determination and line profile fitting. For all sample stars, we assumed identical uncertainties in the effective temperature, surface gravity, and microturbulence velocity, which were $80 \mathrm{~K}, 0.1 \mathrm{dex}$, and $0.1 \mathrm{~km} \mathrm{~s}^{-1}$, respectively. Error of the continuum placement was determined by computing standard deviation of the continuum, $\sigma_{\text {cont }}$, in the vicinity of the investigated spectral lines and then changing continuum level by $\pm 1 \sigma_{\text {cont }}$ from the adopted level and re-determining abundances. The fit error was estimated by computing standard deviation of the difference between the observed and best-fitted spectral line profiles. Again, the synthetic spectrum was changed by $\pm 1 \sigma$ and the resulting difference in the abundance was computed. These individual errors were then added in quadratures and were used further as uncertainties in the determined abundances of $\mathrm{Fe}, \mathrm{Na}$, and $\mathrm{Ba}$. The typical abundance errors are provided in Table 4.

\section{Results and discussion}

\subsection{Possible Ba-Na anti-correlation in the RGB stars in 47 Tuc?}

The barium-to-iron abundance ratios, $[\mathrm{Ba} / \mathrm{Fe}]$, determined in 261 RGB stars show a weak but statistically significant anticorrelation with the sodium-to-iron abundance ratios, $[\mathrm{Na} / \mathrm{Fe}]$ (Fig 5], with the Pearson's correlation coefficient $r_{\mathrm{P}}=-0.18$. Assuming a null-hypothesis that there is no correlation between the two abundance ratios, the Student's $t$-test gives a probability of $p_{\mathrm{P}}=2.9 \times 10^{-3}$ that such $r_{\mathrm{P}}$ value could be obtained by chance (Table 5). The Spearman's rank correlation coefficient and the $p$ value are, respectively, $\rho_{S}=-0.20$ and $p_{\mathrm{S}}=1.3 \times 10^{-3}$, while for the Kendall's rank correlation the two values are $\tau_{K}=-0.14$ and $p_{\mathrm{K}}=6.9 \times 10^{-4}$, respectively.

We also checked whether such anti-correlation may exist between the determined $[\mathrm{Ba} / \mathrm{Fe}]$ abundance ratios and the distance of the target RGB stars from the cluster center. For this, we adopted the coordinates of the 47 Tuc center, 


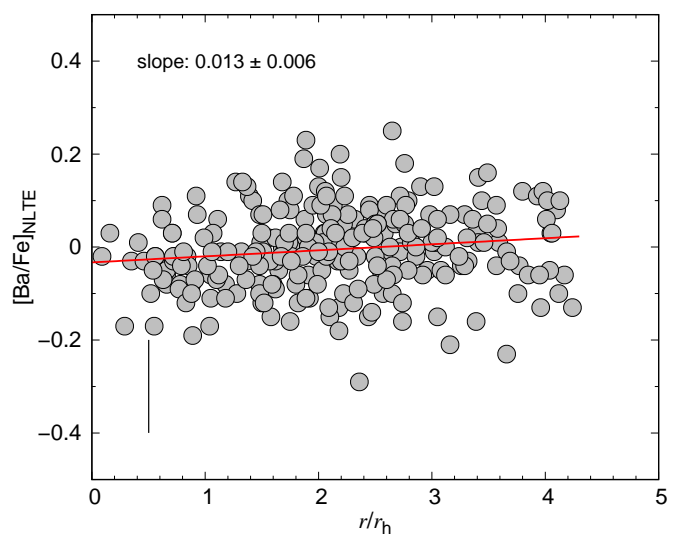

Fig. 6. $[\mathrm{Ba} / \mathrm{Fe}]$ abundance ratios obtained in $261 \mathrm{RGB}$ stars in 47 Tuc, plotted versus relative radial distance from the cluster center. Cluster half-mass radius, $r_{\mathrm{h}}=174$ arcsec, was taken from Trager et al. (1993). Linear fit to the data is marked as the solid red line. Typical abundance error is shown as the vertical bar in the bottom left corner.

Table 5. The $[\mathrm{Ba} / \mathrm{Fe}]-[\mathrm{Na} / \mathrm{Fe}]$ abundance correlation coefficients and their in the full sample of 261 RGB stars in 47 Tuc and sub-samples divided into $\Delta T_{\text {eff }}=100 \mathrm{~K}$-wide bins. $N$ is the number of stars in the given (sub-)sample.

\begin{tabular}{|c|c|c|c|c|c|}
\hline \multicolumn{2}{|c|}{ Pearson } & \multicolumn{2}{|c|}{ Spearman } & \multicolumn{2}{|c|}{ Kendall } \\
\hline$r$ & $p$ & $\rho$ & $p$ & $\tau$ & $p$ \\
\hline \multicolumn{6}{|c|}{ Full sample, $\mathrm{N}=261$} \\
\hline-0.18 & $2.93 \times 10^{-3}$ & -0.20 & $1.30 \times 10^{-3}$ & -0.14 & $6.88 \times 10^{-4}$ \\
\hline \multicolumn{6}{|c|}{$4200 \leq T_{\text {eff }}<4300, \mathrm{~N}=23$} \\
\hline-0.11 & 0.61 & -0.09 & 0.68 & -0.06 & 0.68 \\
\hline \multicolumn{6}{|c|}{$4300 \leq T_{\mathrm{eff}}<4400, \mathrm{~N}=15$} \\
\hline-0.15 & 0.60 & -0.17 & 0.54 & -0.16 & 0.42 \\
\hline \multicolumn{6}{|c|}{$4400 \leq T_{\mathrm{eff}}<4500, \mathrm{~N}=35$} \\
\hline \multicolumn{6}{|c|}{$4500 \leq T_{\text {eff }}<4600, \mathrm{~N}=42$} \\
\hline-0.15 & 0.35 & -0.15 & 0.32 & -0.11 & 0.31 \\
\hline \multicolumn{6}{|c|}{$4600 \leq T_{\text {eff }}<4700, \mathrm{~N}=48$} \\
\hline-0.41 & $3.77 \times 10^{-3}$ & -0.41 & $3.19 \times 10^{-3}$ & -0.28 & $4.87 \times 10^{-3}$ \\
\hline \multicolumn{6}{|c|}{$4700 \leq T_{\text {eff }}<4800, \mathrm{~N}=66$} \\
\hline-0.11 & 0.39 & -0.16 & 0.20 & -0.12 & 0.15 \\
\hline \multicolumn{6}{|c|}{$4800 \leq T_{\text {eff }}<4800, \mathrm{~N}=32$} \\
\hline 0.15 & 0.42 & 0.04 & 0.83 & 0.03 & 0.84 \\
\hline
\end{tabular}

$\alpha_{0}(J 2000)=6.023625 \mathrm{deg}$ and $\delta_{0}(J 2000)=-72.081276 \mathrm{deg}$, from Baumgardt et al. (2019) and the half-mass radius of $r_{\mathrm{h}}=$ 174 arcsec from Trager et al. (1993). Our data suggest a weak correlation in the $[\mathrm{Ba} / \mathrm{Fe}]-r_{\mathrm{h}}$ plane, with $r_{\mathrm{P}}=0.14$ and $p_{\mathrm{P}}=0.019$ (Fig. 6); similar values were obtained for the Spearman's and Kendall's rank correlation coefficients.

On the one hand, these results may suggest that in 47 Tuc the $1 \mathrm{P}$ stars (lower $\mathrm{Na}$ abundances) are more abundant in $\mathrm{Ba}$ than the $2 \mathrm{P}$ stars (higher Na abundances; Fig. 5). Because the 2P stars tend to concentrate towards the cluster center in this and other GGCs (e.g. Kučinskas et al. 2014), this should lead to a weak correlation in the $[\mathrm{Ba} / \mathrm{Fe}]-r_{\mathrm{h}}$ plane. This is exactly what our data suggest (Fig. 6). However, this result would be difficult to explain from the nucleosynthesis point of view. It is well known that $\mathrm{Ba}$ could be synthesised either in the low- and intermediatemass AGB stars via the main s-process or during the central $\mathrm{He}$ -

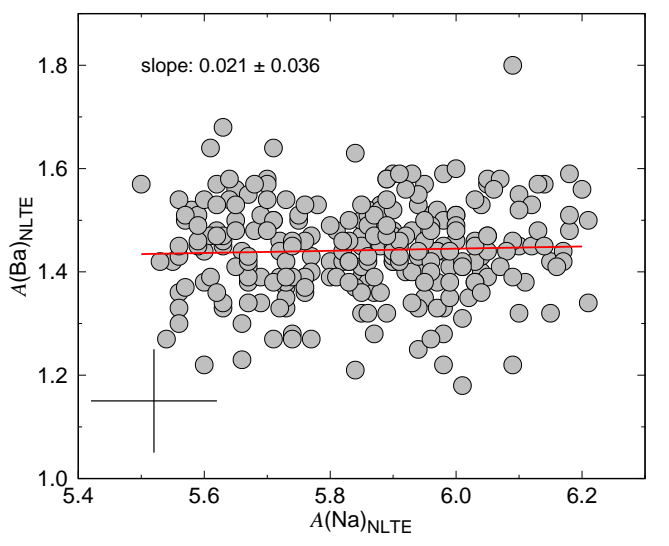

Fig. 7. Absolute abundances of $\mathrm{Ba}$ and $\mathrm{Na}$ in the $A(\mathrm{Ba})-A(\mathrm{Na})$ plane. Typical abundance error bars are shown in the bottom left corner. The linear fit to the data is shown as the solid red line.

burning phase in the massive rotating stars. However, the trends suggested by our data would require a mechanism which would destroy $\mathrm{Ba}$ in the $2 \mathrm{P}$ stars instead of synthesising it like, e.g. Na.

On the other hand, no $\mathrm{Ba}-\mathrm{Na}$ anti-correlation is seen in the absolute abundance plane, $A(\mathrm{Ba})-A(\mathrm{Na})$ (Fig.7). In this case we obtained $r_{\mathrm{P}}=0.04$ and $p_{\mathrm{P}}=0.52$, with similar results also for the Spearman's and Kendall's rank correlation coefficients: $\rho_{S}=$ 0.03 and $p_{\mathrm{S}}=0.63$ for the former, and $\tau_{K}=0.03$ and $p_{\mathrm{K}}=0.53$ for the latter. No trend was detected in the $[\mathrm{Ba} / \mathrm{Fe}]-r_{\mathrm{h}}$ plane either, with $r_{\mathrm{P}}=-0.002 \pm 0.006$ and $p_{\mathrm{P}}=0.984$. This therefore suggests that the anti-correlation in the $[\mathrm{Ba} / \mathrm{Fe}]-[\mathrm{Na} / \mathrm{Fe}]$ plane and correlation in the $[\mathrm{Ba} / \mathrm{Fe}]-r_{\mathrm{h}}$ plane may be caused by the variation in $\mathrm{Fe}$ abundance alone.

A closer inspection of the data has shown that the $\mathrm{Ba}-\mathrm{Na}$ anti-correlation in the $[\mathrm{Ba} / \mathrm{Fe}]-[\mathrm{Na} / \mathrm{Fe}]$ plane has been detectable only in a single effective temperature interval (bin), $4600 \leq T_{\text {eff }}<4700 \mathrm{~K}$, with a possible weaker trend seen also in the $4400 \leq T_{\text {eff }}<4500 \mathrm{~K}$ bin (Table 5). No statistically significant correlation was detected in other $\Delta=100 \mathrm{~K}$ wide effective temperature bins. It therefore seems that the anti-correlation in the $4600 \leq T_{\text {eff }}<4700 \mathrm{~K}$ bin was caused by several outlying stars with the somewhat higher and lower $\mathrm{Ba}$ abundances at the lowest and highest $\mathrm{Na}$ abundance ends, respectively (see Sect. C). No residual correlation is seen if these outliers are excluded.

It is also important to stress that abundances of all there elements analysed in this study show trends with the microturbulence velocity (Fig 4). The most likely explanation for this is that microturbulence velocities were slightly underestimated in the hotter $\left(T_{\mathrm{eff}} \gtrsim 4700 \mathrm{~K}\right)$ stars which are fainter and therefore their spectra are of lower quality. For these stars, a slight overestimate of the continuum during the determination of microturbulence velocities would lead to a systematically larger abundances determined from the weaker Fe I lines with respect to those obtained from the stronger lines. This, in turn, would require a lower microturbulence velocity value to eliminate the trend of iron abundance with the equivalent width. Because the Ba II lines are the strongest of all three elements studied, it is not surprising that there is the strongest trend of abundance versus microturbulence velocity for barium. Thus, overestimated $\mathrm{Ba}$ abundances in stars with the lowest microturbulence velocities (i.e. those with the highest effective temperatures) could also contribute to the spurious $[\mathrm{Ba} / \mathrm{Fe}]-[\mathrm{Na} / \mathrm{Fe}]$ anti-correlation. 
Therefore, the most likely reason for the anti-correlation seen in the $[\mathrm{Ba} / \mathrm{Fe}]-[\mathrm{Na} / \mathrm{Fe}]$ plane and correlation in the $[\mathrm{Ba} / \mathrm{Fe}]-r_{\mathrm{h}}$ plane may be a random fluctuation in $\mathrm{Ba}$ abundances in several stars with the extreme $\mathrm{Na}$ abundances in the $4600 \leq T_{\text {eff }}<4700 \mathrm{~K}$ effective temperature bin. This deviation in a single $T_{\text {eff }}$ bin can not be explained by e.g. the lower quality of the spectra, because for the hotter and therefore fainter stars there is no such correlation. Similarly, there is no plausible astrophysical reason why such correlation should exist in a single $T_{\text {eff }}$ bin only. We also note that no $\mathrm{Ba}-\mathrm{Na}$ correlation was detected by D'Orazi et al. (2010) in their analysis of 110 RGB stars in 47 Tuc which indirectly supports our assertion that the $[\mathrm{Ba} / \mathrm{Fe}]-[\mathrm{Na} / \mathrm{Fe}]$ anti-correlation detected in our study is in fact spurious. This said, it would be nevertheless interesting to see whether the $\mathrm{Ba}-\mathrm{Na}$ - or correlation between any other s-process and light elements - could be seen in other GGCs, especially given the tentative detection of $\mathrm{Zr}-\mathrm{Na}$ correlation in the $\mathrm{RGB}$ stars of 47 Tuc suggested in the analysis of Kolomiecas et al. (2021).

\subsection{Average $\mathrm{Ba}$ abundance in the RGB stars in 47 Tuc}

Until now, Ba abundance in 47 Tuc has been determined in a number of studies. In one of the earlier attempts, James et al. (2004) investigated $\mathrm{Ba}$ in the atmospheres of turn-off (TO) and subgiant (SGB) stars in 47 Tuc. The average Ba LTE abundance determined in eight SGB stars was $[\mathrm{Ba} / \mathrm{Fe}]=+0.35$, with only slightly lower value of $[\mathrm{Ba} / \mathrm{Fe}]=+0.22$ obtained in three TO stars. The average value derived for these stars is therefore slightly larger than the average abundance obtained in our study, given that we determined $\left\langle[\mathrm{Ba} / \mathrm{Fe}]_{1 \mathrm{D} \text { NLTE }}\right\rangle=-0.01 \pm 0.06$ and that the mean 1D NLTE-LTE Ba abundance correction is $\sim-0.10$ dex.

One of the most comprehensive Ba abundance studies in the GGCs was performed by D'Orazi et al. (2010) who studied 1200 stars in 15 GGCs. In case of 47 Tuc they obtained a mean value of $\left\langle[\mathrm{Ba} / \mathrm{Fe}]_{1 \mathrm{D} \text { LTE }}\right\rangle=0.15 \pm 0.06$ in the sample of $110 \mathrm{RGB}$ stars (the error is standard deviation due to star-to-star abundance variation). Again, this value agrees well with that obtained in the present study if the NLTE-LTE correction $(\sim-0.10$ dex $)$ is taken into account. Similarly, a reasonable agreement is obtained with the average Ba LTE abundances obtained by Worley et al. (2010, $[\mathrm{Ba} / \mathrm{Fe}]=0.34 \pm 0.33,3$ RGB stars) and Worley \& Cottrell $(2012,[\mathrm{Ba} / \mathrm{Fe}]=0.32 \pm 0.05,13 \mathrm{RGB}$ stars $)$ if the NLTE-LTE abundance correction is applied.

In their analysis of 13 RGB stars in 47 Tuc Thygesen et al. (2014) determined an average Ba NLTE abundance $[\mathrm{Ba} / \mathrm{Fe}]=$ $0.28 \pm 0.07$ which is significantly higher than that obtained in our study. A possible reason for this discrepancy is differences in the determined surface gravities and microturbulence velocities. Judging from the analysis of 6 stars that were available both in their and our samples we find that on average our microturbulence velocities and gravities were higher by $0.33 \mathrm{~km} / \mathrm{s}$ and 0.3 dex, respectively. Correction for these differences would make our abundances higher by 0.20 dex and would bring them into a good agreement with the value obtained by Thygesen et al. (2014).

\subsection{Implications for the chemical evolution scenarios of 47 Tuc}

Results of Ba abundance analysis in 261 RGB stars suggest that there have been no enrichment or depletion of $\mathrm{Ba}$ in the $2 \mathrm{P}$ stars

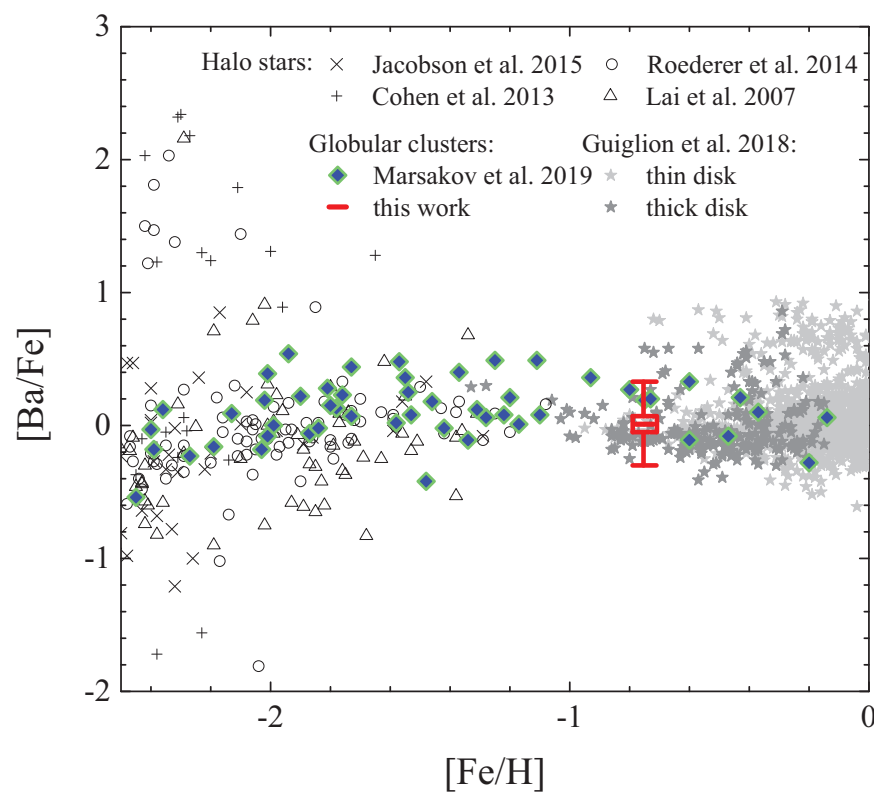

Fig. 8. Abundance of barium in the Galactic globular clusters (compilation by Marsakov et al. (2019)) and Galactic field stars (thin/thick disk stars: Guiglion et al. (2018), halo stars: Lai et al. (2007); Cohen et al. (2013); Roederer et al. (2014); Jacobson et al. (2015)). Average [Ba/Fe] ratio obtained in 261 RGB stars in 47 Tuc is marked by the red symbol where the 25 th, 75 th percentiles and the average values are indicated by the horizontal bars while the extent of the whiskers correspond to the minimum and the maximum abundance values observed in this cluster.

of 47 Tuc. Similarly, no Ba-Na correlation have been detected by D'Orazi et al. (2010) in their analysis of 110 RGB stars. These findings contradict the result obtained by Gratton et al. (2013) who observed a weak but statistically significant $\mathrm{Ba}-\mathrm{Na}$ correlation in the sample of 114 RHB stars in 47 Tuc. However, as was indicated by Grevesse et al. (2015), their detection of a possible $\mathrm{Ba}-\mathrm{Na}$ correlation should be taken with caution because the $[\mathrm{Na} / \mathrm{O}]$ correlation with effective temperature along the $\mathrm{HB}$ could partly or even fully explain the observed weak $\mathrm{Ba}-\mathrm{Na}$ trend.

The obtained average barium-to-iron abundance ratio in 261 RGB stars in 47 Tuc, $\left\langle[\mathrm{Ba} / \mathrm{Fe}]_{1 \mathrm{D} \text { NLTE }}\right\rangle=-0.01 \pm 0.06$, agrees well with the abundances obtained at this metallicity in other GGCs, as well as with those determined in the Galactic disc and halo stars (Fig. 8). This, as well as the relatively narrow spread in Ba abundances of \pm 0.06 dex (which can be fully accounted for by the $\mathrm{Ba}$ abundance determination error, $\sigma_{\mathrm{A}(\mathrm{Ba})} \approx 0.12 \mathrm{dex}$ ), suggest that $\mathrm{Ba}$ abundance in 47 Tuc may reflect that of the primordial proto-cluster gas that has not been altered significantly (if at all) in the subsequent chemical enrichment during the evolution of the GGC.

In a recent analysis of $\mathrm{Zr}$ abundance in $237 \mathrm{RGB}$ stars in 47 Tuc, Kolomiecas et al. (2021) detected a weak but statistically significant $\mathrm{Zr}-\mathrm{Na}$ correlation and $2 \mathrm{P}-1 \mathrm{P} \mathrm{Zr}$ abundance difference of $0.06 \mathrm{dex}$. Assuming that the $2 \mathrm{P}$ were indeed enriched in $\mathrm{Zr}$ but not in Ba would suggest that only some s-process elements have been produced by the polluters that have enriched the $2 \mathrm{P}$ stars in $\mathrm{Na}$ and modified abundances of other light elements such as $\mathrm{Li}, \mathrm{N}, \mathrm{O}, \mathrm{Mg}, \mathrm{Al}$, as determined in the earlier studies (see e.g. Bastian \& Lardo 2018). Theoretical yields from low- and intermediate-mass AGB stars predict that $\mathrm{Zr}$ and $\mathrm{Ba}$ can be produced in substantial amounts but they should be synthesised simultaneously (Cristallo et al. 2015). Similarly, sub- 
stantial amounts of $\mathrm{Zr}$ and $\mathrm{Ba}$ could be produced by explosive nucleosynthesis in massive rotating stars but, again, both elements should be produced simultaneously (Limongi \& Chieffi 2018). Besides, in the latter scenario one would also expect some enrichment in the Fe-group and r-process elements which has not been observed in the Type I GGCs until now (e.g. Bastian \& Lardo 2018; Gratton et al. 2019). It would be therefore very desirable to verify whether correlations between the other s-process and light chemical elements may exist in 47 Tuc as well as in other Type I GGCs.

\section{Conclusions}

We present homogeneous abundances of $\mathrm{Fe}, \mathrm{Na}$, and $\mathrm{Ba}$ obtained in the sample of 261 RGB stars that belong to Galactic globular cluster (GGC) 47 Tuc. Contrary to the earlier finding of Gratton et al. (2013), our results show no statistically significant variation of $\mathrm{Ba}$ abundance with that of $\mathrm{Na}$ thus suggesting that the primordial (Na-poor, $1 \mathrm{P}$ ) and polluted (Na-rich, 2P) populations in this GGC are characterized by the same average $\mathrm{Ba}$ abundance, $[\mathrm{Ba} / \mathrm{Fe}]_{1 \mathrm{D} \text { NLTE }}=0.02 \pm 0.06$ (here the error is standard deviation due to star-to-star abundance variation). This is also supported by the analysis of D'Orazi et al. (2010) who detected no Ba-Na correlation in a sample of 110 RGB stars in this GGC. Taken together with the recent finding of Kolomiecas et al. (2021) who reported a detection of weak but statistically significant $\mathrm{Zr}-\mathrm{Na}$ correlation in $237 \mathrm{RG}$ stars in 47 Tuc, these results would indicate that in this GGC the 2P stars may have been enriched in certain s-process elements (i.e. $\mathrm{Zr}$ ) but not in others (i.e. Ba). It would be therefore very desirable to verify whether such correlations between the abundances of s-process and light chemical elements may exist in other GGCs via the analysis of more s-process elements in large samples of GGC stars, preferably by using spectra of higher resolution and better signal-to-noise ratio.

Acknowledgements. We thank the anonymous referee for useful comments that significantly helped to improve the paper. This study has benefited from the activities of the "ChETEC" COST Action (CA16117), supported by COST (European Cooperation in Science and Technology) and from the European Union's Horizon 2020 research and innovation programme under grant agreement No 101008324 (ChETEC-INFRA). JK acknowledges support from European Social Fund (project No 09.3.3-LMT-K-712-19-0172) under grant agreement with the Research Council of Lithuania (LMTLT). This work has made use of the VALD database, operated at Uppsala University, the Institute of Astronomy RAS in Moscow, and the University of Vienna.

\section{References}

Alonso, A., Arribas, S., \& Martínez-Roger, C. 1999, A\&AS, 140, 261A Andrievsky, S. M., Spite, M., Korotin, S. A., et al. 2009, A\&A, 494, 1083 Barklem, P. S., Belyaev, A. K., Dickinson, A. S., et al. 2010, A\&A, 519, A20 Bastian, N. \& Lardo, C. 2018, ARA\&A, 56, 83

Baumgardt, H., Hilker, M., Sollima, A., \& Bellini, A. 2019, MNRAS, 482, 5138 Bergbusch, P. A., \& Stetson, P. B. 2009, AJ, 138, 1455

Bonifacio, P., Pasquini, L., Molaro, P., et al. 2007, A\&A, 470, 153

Caffau, E., Ludwig, H.-G., Steffen, M., Freytag, B., \& Bonifacio, P. 2011, Solar. Phys., 268, 255

Carlsson, M. 1986, UppOR, 33

Carretta, E., Bragaglia, A., Gratton,R. G., Lucatello, S., Catanzaro, G., et al. 2009, A\&A, 505, 117

Černiauskas, A., Kučinskas, A., Klevas, J., Bonifacio, P., Ludwig, H. -G., et al. 2018, A\&A, 616, 142

Cohen, J. G., Christlieb, N., Thompson, I., et al. 2013, ApJ, 778, 56

Cristallo, S., Straniero, O., Piersanti, L., \& Gobrecht, D. 2015, ApJS, 219, 40

D’Antona, F., Vesperini, E., D'Ercole, A., et al. 2016, MNRAS, 458, 2122

Decressin, T, Charbonnel, C., Siess, L., et al. 2007, A\&A, 505, 727

Denisenkov, P. A., \& Hartwick, F. D. A. 2014, MNRAS, 437, L21
Dobrovolskas, V., Kučinskas, A., Bonifacio, P., Caffau, E., Ludwig, H.-G., et al. 2014, A\&A, 576, 128

D’Orazi, V., Gratton, R., Lucatello, S., Carretta, E., Bragaglia, A., \& Marino, A. F. 2010, ApJL, 719L, 213

D’Orazi, V. D., Campbell, S. W., Lugaro, M., et al. 2013, MNRAS, 433, 366

Doyle, A.P., Davies, G.R., Smalley, B., Chaplin, W.J., Elsworth, Y. 2014, MNRAS, 444, 3592

Gaia Collaboration, Brown, A. G. A., Vallenari, A., et al. 2021, A\&A, 649, A1 Gallagher, A. J., Bergemann, M., Collet, R., et al. 2020, A\&A, 634, A55

Gieles, M., Charbonnel, C., Krause, M. G. H., et al. 2018, MNRAS, 478, 2461

Gratton, R. G., Lucatello, S., Sollima, A., et al. 2013, A\&A, 549, A41

Gratton, R. G., Bragaglia, A., Carretta, E., et al. 2019, Astron. Astrophys. Review, 27,8

Grevesse, N., Scott, P., Asplund M., \& Sauval, A.J. 2015, A\&A, 573, A27

Guiglion, G., de Laverny, P., Recio-Blanco, A., \& Prantzos, N. 2018, A\&A, 619, 143

Jacobson, H. R., Keller, S., Frebel, A., et al. 2015, ApJ, 807, 171

James, G., François, P., Bonifacio, P., Carretta, E., Gratton, R. G., \& Spite, F. 2004, A\&A, 427, 825

Kim, Y.-C., Demarque, P., Yi, S. K., \& Alexander, D. R. 2002, ApJS, 143, 499

Kolomiecas, E., Dobrovolskas, V., Kučinskas, A., Bonifacio, P., \& Korotin, S. 2021, A\&A, submitted.

Korotin, S. A., Andrievsky, S. M., \& Luck, R. E. 1999, A\&A, 351, 168

Korotin, S., Mishenina, T., Gorbaneva, T., \& Soubiran, C. 2011, MNRAS, 415, 2093

Kučinskas, A., Dobrovolskas, V., \& Bonifacio, P. 2014, A\&A, 568, L4

Kupka, F., Ryabchikova, T.A., Piskunov, N.E., Stempels, H.C., \& Weiss, W.W. 2000, BaltA, 9, 590

Kurucz, R. L., Furenlid, I., Brault, J., \& Testerman, L. 1984, Solar Flux Atlas from 296 to 1300nm, National Solar Observatory, Sunspot, New Mexico

Kurucz, R.L. 1993, ATLAS9 Stellar Atmosphere Programs and 2 km/s Grid, CD-ROM No.13, Cambridge, Mass.

Lai, D. K., Johnson, J. A., Bolte, M., et al. 2007, ApJ, 667, 1185

Lodders, K. 2021, Space Sci. Rev., 591, 1220

Limongi, M., Chieffi, A. 2018, ApJS, 237, 13

Marino, A. F., Milone, A. P., Karakas, A. I., et al. 2015, MNRAS, 450, 815

Marino, A. F., Milone, A. P., Renzini, A., et al. 2019, MNRAS, 487, 3815

Marsakov, V. A., Koval, V. V., \& Gozha, M. L. 2019, ARep, 63, 274

Mashonkina, L. I., \& Bikmaev, I. F. 1996, ARep, 40, 94

Milone, A. P., Marino, A. F., Mastrobuono-Battisti, A., \& Lagioia, E. P. 2018, MNRAS, 479, 5005

Pasquini, L., Bonifacio, P., Molaro, P., et al. 2005, A\&A, 441, 549

Ramírez, I., \& Meléndez, J. 2005, ApJ, 626, 465

Roederer, I. U., Preston, G. W., Thompson, I. B., et al. 2014, AJ, 147, 136

Ryabchikova, T., Piskunov, N., Kurucz, R. L., et al. 2015, Phys. Scr., 90, 054005

Sbordone, L., Bonifacio, P., Castelli, F., \& Kurucz, R. L. 2004, Mem. Soc. Astron. Italiana, 5, 93

Sbordone, L. 2005, Mem. Soc. Astron. Italiana, 8, 61

Scott, P., Asplund, M., Grevesse, N., Bergemann, M., \& Sauval, A. J. 2015a, A\&A, 573, A25

Shen, Z.-X., Bonifacio, P., Pasquini, L., et al. 2010, A\&A, 524, L2

Spite, M., Spite, F., Gallagher, A.J., et al. 2016, A\&A, 594, A79

Thygesen, A. O., Sbordone, L., Andrievsky, S., Korotin, S., Yong, D., et al. 2014, A\&A, 572, 108

Tody, D. 1986, Proc. SPIE, 627, 733

Trager, S. C., Djorgovski, S., \& King, I. R. 1993, ASPC, 50, 347

Tsymbal, V. 1996, in M.A.S.S., Model Atmospheres and Spectrum Synthesis, eds. S. J. Adelman, F. Kupka, \& W. W. Weiss, ASP Conf. Ser., 108, 198

Villanova, S., \& Geisler, D. 2011, A\&A, 535, A31

Wiese, W. L., \& Martin, G. A. 1980, in Wavelengths and transition probabilities for atoms and atomic ions: Part 2, Transition probabilities, NSRDS-NBS, Vol. 68

Worley C. C., Cottrell, P. L., McDonald, I., \& van Loon, J. Th. 2010, MNRAS, 402, 2060

Worley, C. C., \& Cottrell, P. L. 2012, PASA, 29, 29 
Table A.1. Target stars common to different observing programs.

\begin{tabular}{|c|c|c|}
\hline 072.D-0777(A) & 073.D-0211(A) & 088.D-0026(A) \\
\hline B-1256 & - & R287 \\
\hline F-1389 & 1389 & - \\
\hline B-3449 & - & R563 \\
\hline F-3476 & - & R317 \\
\hline F-4373 & 4373 & - \\
\hline- & 5172 & $\mathrm{R} 259$ \\
\hline B-5362 & - & $\mathrm{R} 253$ \\
\hline F-7711 & 7711 & - \\
\hline- & 7904 & $\mathrm{R} 443$ \\
\hline B-7993 & - & $\mathrm{R} 277$ \\
\hline B-9163 & - & $\mathrm{R} 800$ \\
\hline- & 9518 & R237 \\
\hline- & 9717 & R682 \\
\hline B-9997 & - & $\mathrm{R} 248$ \\
\hline F-10198 & - & R756 \\
\hline F-10527 & - & $\mathrm{R} 782$ \\
\hline- & 10994 & $\mathrm{R} 795$ \\
\hline F-12408 & - & R784 \\
\hline F-13668 & 13668 & - \\
\hline B-13795 & 13795 & $\mathrm{R} 752$ \\
\hline B-13853 & - & $\mathrm{R} 246$ \\
\hline B-14583 & 14583 & - \\
\hline F-15451 & 15451 & - \\
\hline- & 15552 & R381 \\
\hline- & 16597 & R778 \\
\hline B-16667 & - & $\mathrm{R} 790$ \\
\hline B-17819 & - & $\mathrm{R} 245$ \\
\hline- & 21369 & R249 \\
\hline F-23236 & 23236 & - \\
\hline F-24463 & 24463 & - \\
\hline B-29146 & - & $\mathrm{R} 256$ \\
\hline- & 29490 & $\mathrm{R} 231$ \\
\hline F-30104 & 30104 & - \\
\hline B-30463 & 30463 & - \\
\hline B-30949 & - & R766 \\
\hline B-32730 & 32730 & - \\
\hline B-35878 & 35878 & - \\
\hline B-38976 & - & R762 \\
\hline B-41429 & - & R392 \\
\hline B-42866 & 42866 & R656 \\
\hline B-42887 & - & R760 \\
\hline F-43632 & 43632 & R512 \\
\hline B-43852 & 43852 & R704 \\
\hline B-43889 & 43889 & $\mathrm{R} 450$ \\
\hline
\end{tabular}

\section{Appendix A: Differences between $\mathrm{Ba}$ abundances determined in different observing samples}

As was indicated in Sect. 3, a number of target RGB stars have been observed in several observing programs (Table A.1). A comparison of $\mathrm{Fe}, \mathrm{Na}$, and $\mathrm{Ba}$ abundances obtained using spectra that were acquired in the different programs is provided in Fig. A.1 A.3. In most cases, differences between the average abundances obtained in various samples was very small and did not exceed 0.03 dex (Figs A.1 A.2).

The final abundances of the target RGB stars were further corrected for these small systematic shifts. Since the program 072.D-0777(A) had the largest number of stars, we chose to apply abundance shifts relative to this sample. For stars in 073.D-

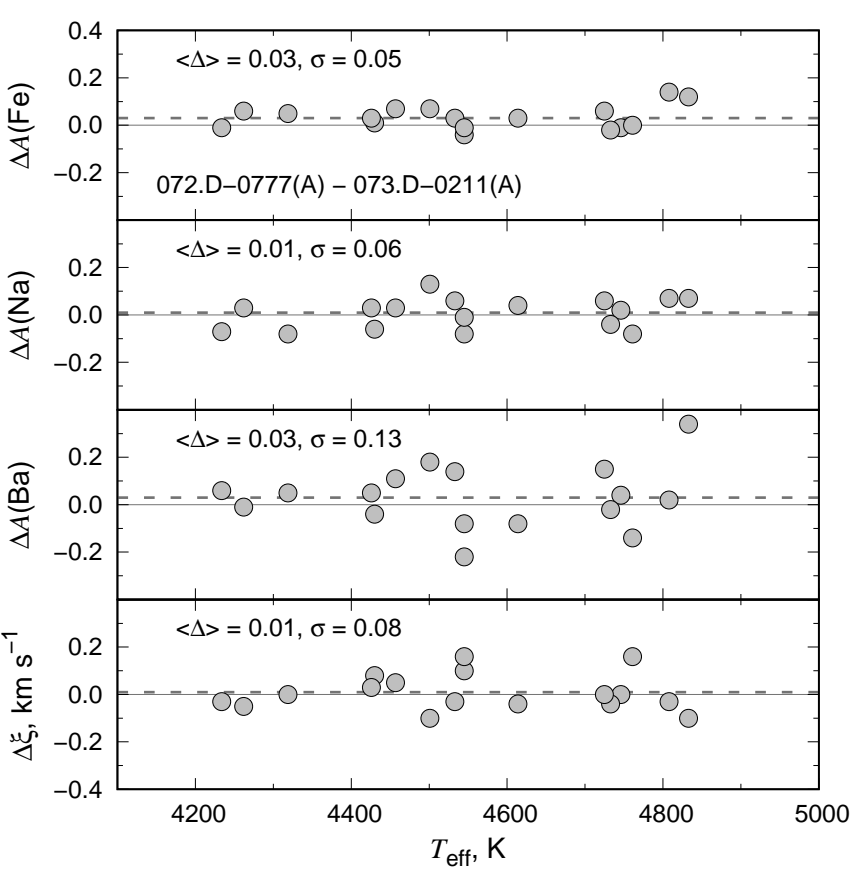

Fig. A.1. Comparison of the abundances (top three pannels) and microturbulence velocity values between the common stars in the programs 072.D-0777(A) and 073.D-0211(A). Grey dashed line shows average value of the difference.

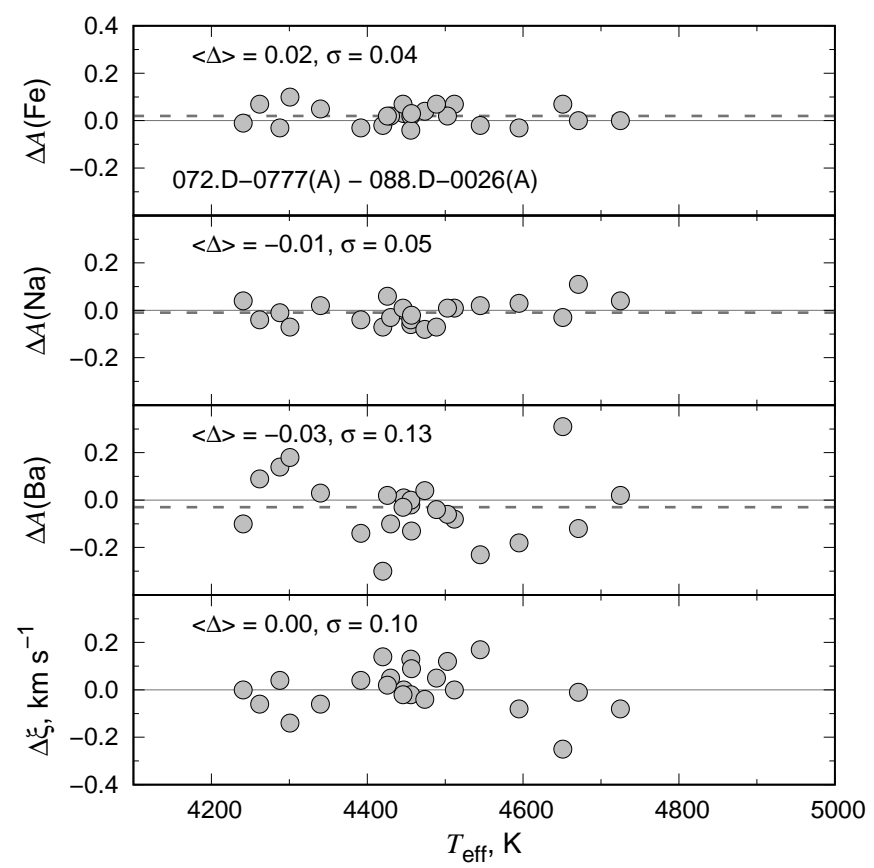

Fig. A.2. Comparison of the abundances (top three pannels) and microturbulence velocity values between the common stars in the programs 072.D-0777(A) and 088.D-0026(A). Grey dashed line shows average value of the difference.

0211(A) sample we applied abundance shifts of $+0.03,+0.01$, +0.03 dex for the abundances of $\mathrm{Fe}, \mathrm{Na}$, and $\mathrm{Ba}$, respectively. In case of the sample 088.D-0026(A), abundances of Fe, $\mathrm{Na}$, and Ba were corrected by $+0.02,-0.01$, and -0.03 dex, respectively. The final abundance ratios (Table D.1) were computed using Solar reference values derived in Sect. 3.1 . 


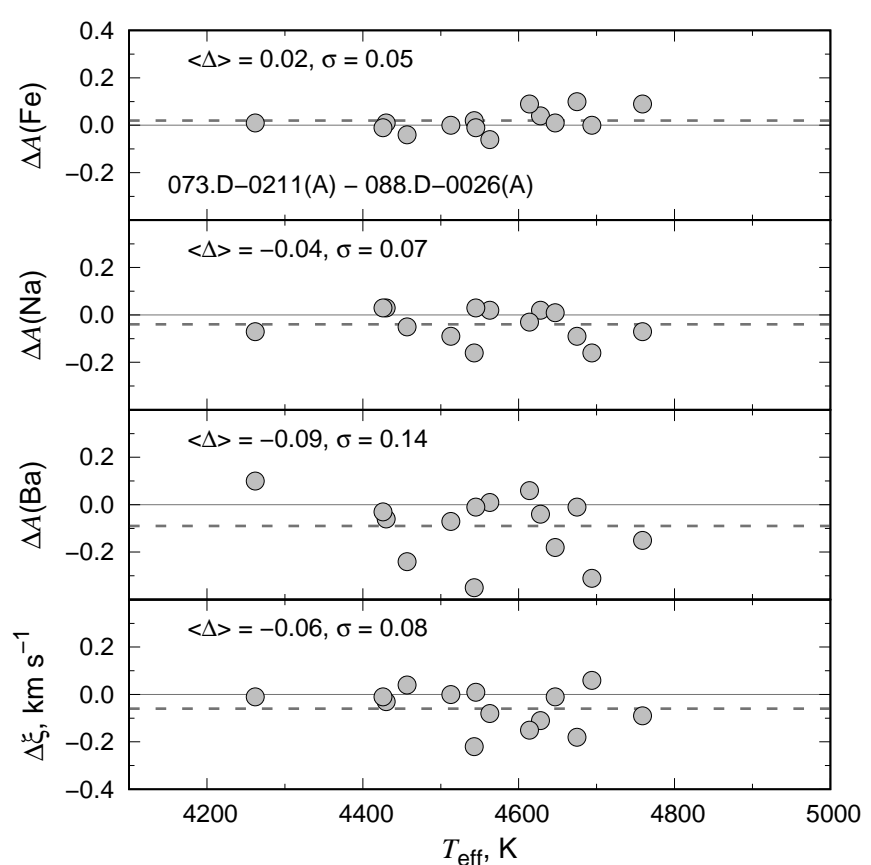

Fig. A.3. Comparison of the abundances (top three pannels) and microturbulence velocity values between the common stars in the programs 073.D-0211(A) and 088.D-0026(A). Grey dashed line shows average value of the difference.

\section{Appendix B: The Fe I and Fe II lines used in the abundance analysis}

The list of Fe I and Fe II lines and their atomic parameters is provided in the Table B.1. We note that abundances from Fe II lines were only determined with the purpose of checking the agreement between the surface gravities obtained from photometry and those derived using Fe II lines; however, they were not used in further analysis. Thus, $\mathrm{Fe}$ abundances used throughout this paper are those that were obtained using Fe I lines.

\section{Appendix C: Abundances of $\mathrm{Ba}$ in the target RGB stars with $4600<T_{\text {eff }}<4700 \mathrm{~K}$}

As noted in Sect. 4, our data hint to a weak $\mathrm{Ba}-\mathrm{Na}$ anticorrelation. Further analysis has revealed that this may be an artefact caused by several spurious outliers with $4600<T_{\text {eff }}<$ $4700 \mathrm{~K}$ that were observed in one of the programs, 073.D0211(A) (Fig.C.1). No such anti-correlation is seen in any other $\Delta T_{\text {eff }}=100 \mathrm{~K}$ wide effective temperature bins. Indeed, no statistically significant anti-correlation is left in the $4600<T_{\text {eff }}<$ $4700 \mathrm{~K}$ either when stars 19992, 38289, and 21369 are excluded. We therefore conclude that there is no statistically significant $\mathrm{Ba}-\mathrm{Na}$ correlation in the analysed sample of $261 \mathrm{RGB}$ stars in 47 Tuc.

\section{Appendix D: Abundances of $\mathrm{Fe}, \mathrm{Na}$, and $\mathrm{Ba}$ in 261 target RGB stars in 47 Tuc}

Abundances of Fe (LTE), Na (NLTE), and Ba (NLTE) obtained in 261 RGB stars in 47 Tuc are provided in Table D.1
Table B.1. The list of iron spectral lines used in the abundance determination.

\begin{tabular}{cccc}
\hline \hline$\lambda, \mathrm{nm}$ & $\chi, \mathrm{eV}$ & $\log g f$ & Ion. $_{\mathrm{stage}_{\mathrm{I}}}$ \\
\hline 612.79070 & 4.140 & -1.398 & $\mathrm{Fe}_{\mathrm{I}}$ \\
615.16180 & 2.180 & -3.300 & $\mathrm{Fe}_{\mathrm{I}}$ \\
616.53600 & 4.140 & -1.460 & $\mathrm{Fe}_{\mathrm{I}}$ \\
617.33360 & 2.220 & -2.810 & $\mathrm{Fe}_{\mathrm{I}}$ \\
618.02040 & 2.730 & -2.650 & $\mathrm{Fe}_{\mathrm{I}}$ \\
618.79900 & 3.940 & -1.580 & $\mathrm{Fe}_{\mathrm{I}}$ \\
620.03130 & 2.610 & -2.310 & $\mathrm{Fe}_{\mathrm{I}}$ \\
621.34300 & 2.220 & -2.550 & $\mathrm{Fe}_{\mathrm{I}}$ \\
621.92810 & 2.200 & -2.410 & $\mathrm{Fe}_{\mathrm{I}}$ \\
622.92283 & 2.845 & -2.805 & $\mathrm{Fe}_{\mathrm{I}}$ \\
623.26410 & 3.650 & -1.130 & $\mathrm{Fe}_{\mathrm{I}}$ \\
624.63188 & 3.602 & -0.733 & $\mathrm{Fe}_{\mathrm{I}}$ \\
625.25554 & 2.404 & -1.687 & $\mathrm{Fe}_{\mathrm{I}}$ \\
626.51340 & 2.180 & -2.510 & $\mathrm{Fe}_{\mathrm{I}}$ \\
627.02250 & 2.860 & -2.570 & $\mathrm{Fe}_{\mathrm{I}}$ \\
627.12788 & 3.332 & -2.703 & $\mathrm{Fe}_{\mathrm{I}}$ \\
630.15012 & 3.654 & -0.718 & $\mathrm{Fe}_{\mathrm{I}}$ \\
631.58115 & 4.076 & -1.710 & $\mathrm{Fe}_{\mathrm{I}}$ \\
632.26860 & 2.590 & -2.280 & $\mathrm{Fe}_{\mathrm{I}}$ \\
633.53308 & 2.198 & -2.177 & $\mathrm{Fe}_{\mathrm{I}}$ \\
633.68243 & 3.686 & -0.856 & $\mathrm{Fe}_{\mathrm{I}}$ \\
634.41490 & 2.430 & -2.890 & $\mathrm{Fe}_{\mathrm{I}}$ \\
638.07430 & 4.190 & -1.270 & $\mathrm{Fe}_{\mathrm{I}}$ \\
660.91100 & 2.560 & -2.610 & $\mathrm{Fe}_{\mathrm{I}}$ \\
670.35670 & 2.760 & -3.010 & $\mathrm{Fe}_{\mathrm{I}}$ \\
672.66660 & 4.610 & -1.010 & $\mathrm{Fe}_{\mathrm{I}}$ \\
675.01530 & 2.420 & -2.580 & $\mathrm{Fe}_{\mathrm{I}}$ \\
680.68450 & 2.730 & -3.090 & $\mathrm{Fe}_{\mathrm{I}}$ \\
681.02630 & 4.610 & -0.940 & $\mathrm{Fe}_{\mathrm{I}}$ \\
684.36560 & 4.550 & -0.780 & $\mathrm{Fe}_{\mathrm{I}}$ \\
685.51620 & 4.560 & -0.570 & $\mathrm{Fe}_{\mathrm{I}}$ \\
685.81500 & 4.610 & -0.910 & $\mathrm{Fe}_{\mathrm{I}}$ \\
691.66820 & 4.150 & -1.260 & $\mathrm{Fe}_{\mathrm{I}}$ \\
614.92580 & 3.890 & -2.720 & $\mathrm{Fe}_{\mathrm{II}}$ \\
623.83920 & 3.870 & -2.520 & $\mathrm{Fe}_{\mathrm{II}}$ \\
624.75570 & 3.890 & -2.320 & $\mathrm{Fe}_{\mathrm{II}}$ \\
& 2.891 & -4.160 & $\mathrm{Fe}_{\mathrm{II}}$ \\
\hline
\end{tabular}




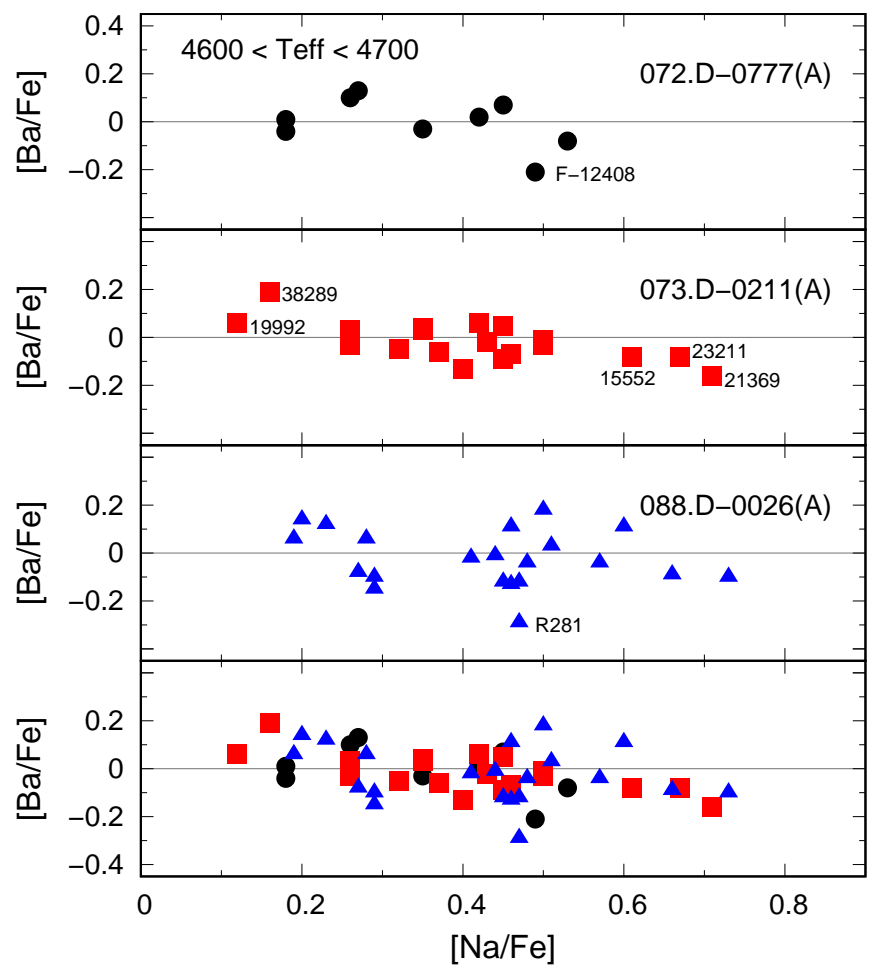

Fig. C.1. Barium and sodium abundances in the effective temperature range $4600<T_{\text {eff }}<4700 \mathrm{~K}$. Labels next to the outlying data points show identifications of the stars. 
Table D.1. Abundances of $\mathrm{Fe}, \mathrm{Na}$, and Ba determined in the sample of 261 stars in 47 Tuc. In cases when abundances were determined using data from several observing programs, microturbulence velocities and abundances obtained using spectra from each individual program are provided. The IDs of individual stars and IDs of the corresponding observing programs are given in the last two columns, respectively (072.D-0777(A), PI: P. François; 073.D-0211(A), PI: E. Carretta; 088.D-0026(A), PI: I. McDonald). The complete table is available in electronic form.

\begin{tabular}{cccccccccccc}
\hline \hline GAIA Source ID & $\begin{array}{c}T_{\text {eff }} \\
\mathrm{K}\end{array}$ & $\log g$ & $\begin{array}{c}\xi_{\text {micro }} \\
\mathrm{km} \mathrm{s}^{-1}\end{array}$ & $A(\mathrm{Fe})$ & {$[\mathrm{Fe} / \mathrm{H}]$} & $A(\mathrm{Na})$ & {$[\mathrm{Na} / \mathrm{Fe}]$} & $A(\mathrm{Ba})$ & {$[\mathrm{Ba} / \mathrm{Fe}]$} & ID & Obs. program \\
\hline 4689575185928019840 & 4275 & 1.45 & 1.47 & 6.77 & -0.78 & 5.58 & 0.19 & 1.54 & 0.15 & R761 & $088 . \mathrm{D}-0026(\mathrm{~A})$ \\
4689575289007212416 & 4231 & 1.34 & 1.56 & 6.77 & -0.78 & 5.60 & 0.21 & 1.54 & 0.15 & R772 & $088 . \mathrm{D}-0026(\mathrm{~A})$ \\
4689581263316313856 & 4553 & 2.07 & 1.53 & 6.77 & -0.78 & 5.63 & 0.24 & 1.39 & 0.00 & R786 & $088 . \mathrm{D}-0026(\mathrm{~A})$ \\
4689613840634674048 & 4271 & 1.48 & 1.48 & 6.82 & -0.73 & 5.66 & 0.22 & 1.38 & -0.06 & 5277 & $073 . \mathrm{D}-0211(\mathrm{~A})$ \\
4689614837066802944 & 4630 & 2.09 & 1.40 & 6.79 & -0.76 & 5.84 & 0.43 & 1.28 & -0.13 & 6808 & $073 . \mathrm{D}-0211(\mathrm{~A})$ \\
4689614970198135296 & 4245 & 1.34 & 1.57 & 6.78 & -0.77 & 5.56 & 0.16 & 1.36 & -0.04 & B-13396 & $072 . \mathrm{D}-0777(\mathrm{~A})$ \\
4689618238680828800 & 4303 & 1.50 & 1.58 & 6.75 & -0.80 & 5.63 & 0.26 & 1.33 & -0.04 & B-9254 & $072 . \mathrm{D}-0777(\mathrm{~A})$ \\
4689618891515852160 & 4704 & 2.19 & 1.44 & 6.81 & -0.74 & 6.10 & 0.67 & 1.42 & -0.01 & R198 & $088 . \mathrm{D}-0026(\mathrm{~A})$ \\
4689619166393741824 & 4512 & 1.96 & 1.46 & 6.82 & -0.73 & 5.73 & 0.29 & 1.36 & -0.08 & R199 & $088 . \mathrm{D}-0026(A)$ \\
4689621709015302400 & 4560 & 2.04 & 1.29 & 6.81 & -0.74 & 5.88 & 0.45 & 1.42 & -0.01 & R677 & $088 . D-0026(A)$ \\
$\ldots$ & $\ldots$ & $\ldots$ & $\ldots$ & $\ldots$ & $\ldots$ & $\ldots$ & $\ldots$ & $\ldots$ & $\ldots$ & $\ldots$ & $\ldots$ \\
\hline
\end{tabular}

